\title{
Red List of Estonian lichens: revision in 2019
}

\section{Piret Lóhmus ${ }^{1}$, Liis Marmor ${ }^{2}$, Inga Jüriado ${ }^{1}$, Ave Suija ${ }^{1,3}$, Ede Oja ${ }^{1}$, Polina Degtjarenko ${ }^{1,4}$, Tiina Randlane ${ }^{1}$}

\author{
${ }^{1}$ University of Tartu, Institute of Ecology and Earth Sciences, Lai 40, 51005 Tartu, Estonia. \\ E-mail: piret.lohmus@ut.ee \\ ${ }^{2}$ E-mail: liis.marmor@gmail.com \\ ${ }^{3}$ University of Tartu, Natural History Museum, Vanemuise 46, 51014 Tartu, Estonia \\ ${ }^{4}$ Swiss Federal Research Institute WSL, Biodiversity and Conservation Biology, Zürcherstrasse 111, \\ 8903 Birmensdorf, Switzerland
}

\begin{abstract}
The second assessment of the threat status of Estonian lichens based on IUCN system was performed in 2019. The main basis for choosing the species to be currently assessed was the list of legally protected lichens and the list of species assigned to the Red List Categories RE-DD in 2008. Species that had been assessed as Least Concern (LC) in 2008 were not evaluated. Altogether, threat status of 229 lichen species was assessed, among them 181 were assigned to the threatened categories (CR, EN, VU), while no species were assigned to the LC category. Compared to the previous red list, category was deteriorated for $58 \%$ and remained the same for $32 \%$ of species. In Estonia, threatened lichens inhabit mainly forests (particularly dry boreal and nemoral deciduous stands), alvar grasslands, sand dunes and various saxicolous habitats. Therefore, the most frequent threat factors were forest cutting and overgrowing of alvars and dunes (main threat factor for 96 and 70 species, respectfully).
\end{abstract}

Kokkuvôte: 2019. aastal viidi läbi teistkordne IUCN süsteemil póhinev Eesti samblike ohustatuse hindamine. Hinnati liike, mis on riiklikult kaitstud ning liike, mis 2008. aasta hindamise järgi olid Eestis kas regionaalselt välja surnud, kriitilises seisundis, väljasuremisohus, ohualtid, ohulähedased või puuduliku andmestikuga (kategooriad RE-DD). 2008. a-l kategooriasse Soodsas seisundis (LC) kuulunud liike ei kaasatud uude hindamisse. Kokku hinnati 229 samblikuliigi ohustatust, nendest 181 kuulusid ohustatud kategooriatesse (CR, EN vôi VU); ükski hinnatud liik ei sobinud kategooriasse LC. Vôrreldes eelmise punase nimestikuga tôusis kategooria 58\% liikidel ning jäi samaks 32\%-l. Ohustatud samblikuliigid asustavad Eestis peamiselt metsi (eriti palu- ja salumetsi), loopealseid, liivikuid ning erinevaid kivi-kasvupindu. Seetôttu on sagedasemad ohutegurid metsaraie ning loodude ja liivikute kinnikasvamine (oluline tegur vastavalt 96 ja 70 liigile).

Keywords: IUCN, regional red-listing, status deterioration, threat factors

\section{INTRODUCTION}

Since 1979, five Red Lists have been compiled in Estonia: in 1979, 1988, 1998, 2008 and 2018-2019. Lichens (40 species) were included for the first time into the second Red List in 1988 (Anonymous, 1993). Conventional system of threat categories and intuitive-emotional criteria for defining these categories were used in three earlier Red Lists. IUCN categories and strictly established criteria (Standards and Petitions Working Group, 2006) were applied for the first time in the fourth Red List (Tartes, 2010). The according part of the Estonian Red List containing lichens was prepared between 2006 and 2008 (Randlane et al., 2008). At that time, the checklist of Estonian lichens - basis for the assessments - included 1,019 species (Randlane et al., 2006). Out of them, 45.5\%, mainly macrolichens, were evaluated. Among evaluated species,
213 were assigned to one of the following IUCN categories: Regionally Extinct (RE), Critically Endangered (CR), Endangered (EN), Vulnerable (VU), Near Threatened (NT) or Data Deficient (DD); assessment results are publicly available (Table 2 in Randlane et al., 2008). The second assessment of the threat status of Estonian lichens based on IUCN system was performed in 2019. The aim of this article is to present the data of the revised Red List of lichens in Estonia, to discuss the changes compared to the previous red list and to summarize the information about threat factors and habitats of threatened lichens.

\section{MATERIAL AND METHODS}

The main basis for choosing the species to be currently assessed was the list of legally pro- 
tected lichens in Estonia (Keskkonnaministri määrus nr 51, 2004; Vabariigi Valitsuse määrus nr 195, 2004) and the list of species assigned to the IUCN categories RE, CR, EN, VU, NT and DD in 2008 (Table 2, Randlane et al., 2008). In addition to these 215 species, 25 macrolichen species were evaluated for the first time: 12 species which were not assessed in 2008 because of identification difficulties and/or limited data (e.g., species of the Cladonia chlorophaea group, some taxa from the former genera Collema and Leptogium) and 13 species that were recorded in Estonia for the first time within 2008-2018. The species that had been assessed as Least Concern in 2008 (251, except for two protected species) were not evaluated during the current assessment process because of financial and time limitations.

The first author (PL) passed the IUCN Red List Accessor Training Workshop (2015 in Tallinn) and, prior to the project, she introduced the criteria and how they should be applied to the group of lichen experts. The latest IUCN guidelines (ver. 13) for using the Red List categories and criteria (IUCN Standards and Petitions Subcommittee, 2017) were applied, but also supplementary guidelines for applying IUCN criteria for fungi (Dahlberg \& Mueller, 2011) were used. Down-listing of the category (because immigration from outside the region will tend to decrease extinction risk within the region) was not done since there are no data about the immigration possibilities of lichen species from neighbouring countries (see also explanation in Ingerpuu et al., 2018).

The following IUCN categories were used: Regionally Extinct (RE), Critically Endangered (CR), Endangered (EN), Vulnerable (VU), Near Threatened (NT), Data Deficient (DD), Least Concern (LC), Not Applicable (NA) and Not Evaluated (NE). Of them, CR, EN and VU are 'threatened categories' according to the IUCN guidelines. Similarly to the previous assessment in 2008, the species that had not been reliably reported from Estonia after 1950 were considered to be RE. During the evaluation process, applicability of IUCN criteria A (Population Size Reduction; only A2-A4 were considered while criterion A1 was not intendedly used), B (Geographic Range), C (Small Population Size and Decline) and D (Very Small or Restricted Population) was assessed for each species and the according category was assigned to the species. Criterium E (Quantitative Analysis) was not used due to the insufficiency of data. Threshold levels of categories for criteria $B$ and D were not modified. If useful, experts applied quantitative threshold level for category NT provided in Dahlberg \& Mueller (2011).

The length of a generation was considered 17 years for most of species except 7 years for ephemeric species Scytinium schraderi. The term "mature individual of a lichen" follows the concept of "functional individual" sensu Hallingbäck (2007). The number of mature individuals (used in criteria C and D1) was estimated only if suitable data were available, for example, when lichen thalli had systematically been counted or estimated during the methodical surveys. The population was considered to be severely fragmented if the distance between species localities was more than 50 or $100 \mathrm{~km}$ (for vegetatively or spore-dispersed species, respectively). Applying criteria A2 and A3, we estimated, inferred or suspected decline of populations within the time length of three generations (i.e. 51 years); applying criterium A4, in some cases (e.g. Cladonia foliacea and Enchylium tenax), the time length was up to 100 years. The species which taxonomy has been changed (for example, they had been synonymized) since the last Red List, were either evaluated in the usual way within the current species (like Vulpicida tubulosus within V. juniperinus; Saag et al., 2014 ) or they were not evaluated in this project as the current species belonged to LC in 2008 (like Usnea diplotypus within U. dasopoga; Mark et al., 2016).

The main data source for species occurrence records was the PlutoF biodiversity database (https://plutof.ut.ee/; Abarenkov et al., 2010), that incorporates the data of specimens from all main Estonian herbaria (TALL, TAM and TU), numerous literature records and the data of protected lichen species from the Estonian Nature Information System (http://www.eelis. ee). In addition, the datasets of 26 published and unpublished lichen research projects or inventories in Estonia during 2008-2018 were uploaded to the PlutoF database and made available for the expert group. Species assessment sheets together with supporting documentation are available to the registered users of the Estonian Nature Information System. 


\section{RESULTS AND DISCUSSION}

During the recent evaluation in Estonia, the threat status of 229 species was assessed (Table 1). These form $19.4 \%$ of the 1,179 species of the lichenized, lichenicolous and saprophytic fungi (i.e. species traditionally treated by lichenologists) that are currently known in Estonia (Randlane et al., 2018). However, the majority of evaluated species represented lichens, and only one saprotrophic fungus (Arthopyrenia cinereopruinosa) belonged to the list of assessed taxa; no lichenicolous fungi were evaluated. Among the assessed species, 181 were assigned to the threatened categories (CR, EN, VU) while no species were assigned to the LC category (Table 1 and Supplement 1). All legally protected lichen species in Estonia (51 taxa) belong either to the threatened categories or they were assessed as NT.

Table 1. Number of lichen species in the IUCN Red List categories according to the assesments in 2008 and 2019. For abbreviations of categories see Material and Methods.

\begin{tabular}{lll}
\hline Category & 2008 & 2019 \\
\hline RE & 29 & 22 \\
CR & 13 & 64 \\
EN & 32 & 74 \\
VU & 68 & 43 \\
NT & 42 & 20 \\
DD & 29 & 6 \\
LC & 251 & 0 \\
Total & 464 & 229 \\
\hline NA & not applied & 7 \\
NE & 555 & 943 \\
\hline Total number of recorded species & $1019^{*}$ & $1179^{* *}$ \\
* Randlane et al., 2006 & & \\
** Randlane et al., 2018 & &
\end{tabular}

Out of 213 species that were evaluated in 2008 to the categories RE-DD and planned to be newly evaluated in 2019 , eleven taxa appeared not applicable for the new assessment against criteria or, in some cases, it was intentionally decided not to re-evaluate them (Figure 1; see Supplement 2 for explanations). For the rest of the species, the red-listed category deteriorated for $58 \%$ and remained the same for $32 \%$ of the species during the second assessment (Figure 1 ). The reason of deterioration of the category (mainly from VU to EN or CR, and from NT to VU or EN) was, in some extent, related to the wider usage of the criteria $\mathrm{C}$ and $\mathrm{D}$ during this evaluation process; furthermore, the application of the criterium D2 was refined during the current evaluation by considering the risk of taxa to become highly threatened within a very short time. However, the small population size and decline of these species was mainly caused by the degradation of their natural habitats. This was also the reason for several other species whose status has deteriorated. For example, Flavocetraria nivalis that inhabits mainly alvar grasslands in Estonia, has moved from NT category in 2008 to CR in 2019 due to the cessation of traditional land use practices, but also environmental stress and natural disturbances (Leppik et al., 2013). Extensive encroachment of alvar grasslands with tall herbs and shrubs is the main threat also for other epigeic lichens (Leppik et al., 2013, 2015) and hence, for example, 20\% decline of the population within two generations was projected for Solorina saccata (i.e. change of threat category from NT in 2008 to EN in 2019). The status of many old-growth forest specialists (e.g. Alectoria sarmentosa, Leptogium saturninum, Lobaria pulmonaria, Menegazzia terebrata and Megalaria grossa) changed from NT category in 2008 to a threat category in 2019 , mainly because of estimated or projected decline of populations caused by continuous destructive forest management with clear cutting and shortened rotation periods. The decline in

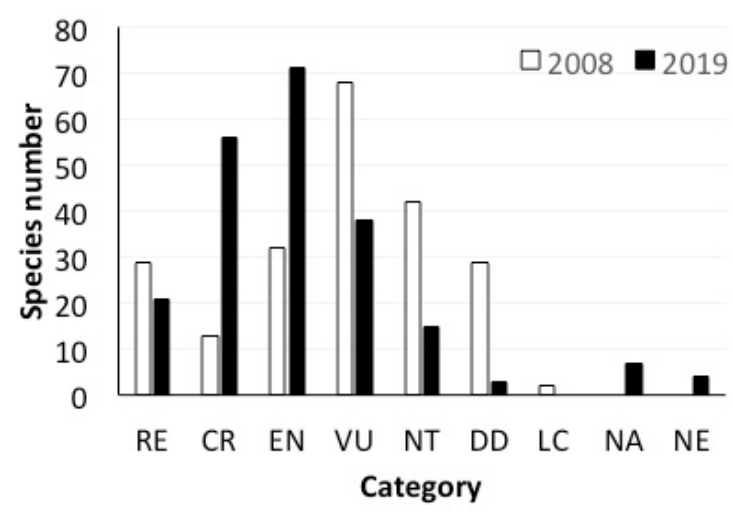

Fig. 1. Distribution of the lichen species that were evaluated both in 2008 and 2019 (n=215) among the IUCN categories. For abbreviations of categories see Material and Methods. 
quantity and quality of forest habitats has been demonstrated in detail particularly for Lobaria pulmonaria (Jüriado \& Liira, 2009, 2010).

The number of RE species is now lower, compared to the previous assessment, as seven species (Diplotomma lutosum, Lecanora epibryon, Peltigera elisabethae, Ramalina elegans, Rostania occultata, Thelidium pyrenophorum and Verrucaria maculiformis) have been re-found within the last ten years. However, there are 14 species that are candidates to be assessed as $\mathrm{RE}$ in the near future, as their last documented occurrence falls to the period of 50-62 years ago (Supplement 1).

In Estonia, threatened lichens inhabit mainly forests (particularly dry boreal and nemoral deciduous stands), alvar grasslands and various saxicolous habitats, but also wooded meadows, parks and limestone quarries and dunes (Figure 2). The wooded habitats provide several specific microhabitats for epiphytic and wood-dwelling lichens, like rough bark of coniferous and deciduous trees, wood of standing and laying tree trunks, charred wood, etc. (e.g. Jüriado et al., 2003; Lõhmus \& Kruustük, 2010; Lõhmus \& Lõhmus, 2011; Degtjarenko et al., 2016; Marmor et al., 2017; Jüriado \& Paal, 2019), while dry alvar grasslands with very thin soil layer and sand dunes are suitable for ground layer community taxa (e.g. Leppik et al. 2013, 2015; Jüriado et al., 2016). Therefore, the main threat factors of Estonian lichens tend to be forest cutting (important factor for 96 species) and overgrowing of dunes and alvars due to the cessation of traditional management (important for 70 species). Furthermore, the air pollution, expansion of urban areas and tourism activity also have negative impact for several lichen species.

The current paper reveals the results of the second assessment of the threat status of Estonian lichens based on IUCN system performed in 2019. However, the species that were assessed as LC in 2008 were not re-evaluated this time (except two protected species), and $59 \%$ of lichens and related fungi currently known in Estonia have not been evaluated with IUCN system at all. Thus, the evaluation process to define the threat status of lichens in Estonia is continuing. Forest microlichens (ca 350 species) will be assessed according to the IUCN categories and criteria during the project "Conservation status of forest lichens in Estonia" in 2020. For this purpose, additional systematic lichen surveys (following method by Lõhmus et al., 2018) will be performed in old-growth stands in less studied regions (islands Saare- and Hiiumaa) and forest site types (alvar forests). Another project, "Contemporary state and threat evaluation of common lichens in Estonia", will provide the assessment of 165 widely distributed lichen species according to IUCN system in 2021.

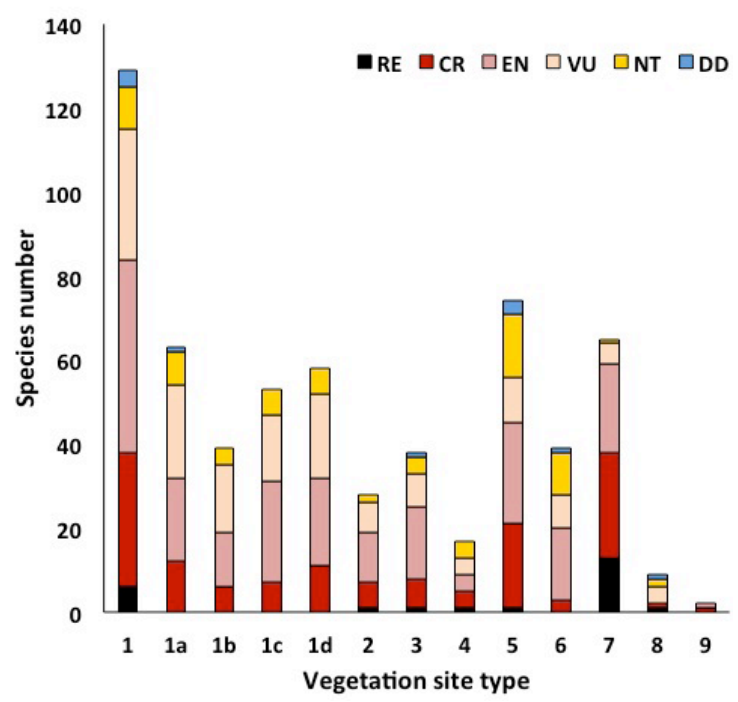

Fig. 2. Distribution of evaluated lichen species (categories RE-DD) among inhabited habitats, based on vegetation site type classification by Paal (1997): 1 - forests (all site types together and more specifically forest of $1 \mathrm{a}$-alvar, boreal heath and dry boreal; $1 \mathrm{~b}$ - fresh boreal and dry boreo-nemoral; 1c - fresh boreo-nemoral; 1d floodplain, paludified and drained peatland); 2 - wooded meadows; 3 - parks; 4 - yards, roadsides, waste grounds; 5 - eutrophic alvar grasslands; 6 - quarries (mainly limestone) and dunes; 7 - outcrops (lime- and sandstone) and erratic boulders; 8 - mires; 9 - fresh waterbodies. Note that one species can inhabit several suitable habitats.

\section{ACKNOWLEDGEMENTS}

We are grateful to Mari Müür for the technical assistance during the evaluation process, to Ljudmilla Martin for making her unpublished data available for us and to the anonymous 
referee for helpful corrections and comments. The project was financed by the Environmental Investment Centre (KIK), the Estonian Research Council (projects IUT 34-7, IUT 20-30, PUT 1017), Mobilitas Pluss programme (MOBTP66) supported by Estonian Research Council and European Regional Development Fund (Centre of Excellence EcolChange).

\section{REFERENCES}

Abarenkov, K., Tedersoo, L., Nilsson, R. H., Vellak, K., Saar, I., Veldre, V., Parmasto, E., Prous, M., Aan, A., Ots, M., Kurina, O., Ostonen, I., Jõgeva, J., Halapuu, S., Põldmaa, K., Toots, M., Truu, J., Larsson, K-H. \& Kõljalg, U. 2010. PlutoF - a Web Based Workbench for Ecological and Taxonomic Research, with an Online Implementation for Fungal ITS Sequences. Evolutionary Bioinformatics 6: 189-196. https:// doi.org/10.4137/EBO.S6271

Anonymous, 1993. Eesti punase raamatu taimed, loomad ja seened (november 1990). In: Eesti A \& O. Tallinn, 1k. 54-60. (In Estonian).

Clerc, P. 2011. Usnea. In: Thell, A. \& Moberg, R. (eds) Nordic Lichen Flora 4: 107-127.

Dahlberg, A. \& Mueller, G. M. 2011. Applying IUCN red-listing criteria for assessing and reporting on the conservation status of fungal species. Fungal Ecology 4(2): 147-162. https://doi. org/10.1016/j.funeco.2010.11.001

Degtjarenko, P., Marmor, L. \& Randlane, T. 2016. Changes in bryophyte and lichen communities on Scots pines along an alkaline dust pollution gradient. Environmental Science and Pollution Research 23: 17413-17425. https://doi.org/ 10.1007/s11356-016-6933-5

Hallingbäck, T. 2007. Working with Swedish cryptogam conservation. Biological Conservation 135: 311-314. https://doi.org/10.1016/j. biocon.2006.10.019

Ingerpuu, N., Vellak, K. \& Ehrlich, L. 2018. Revised Red Data List of Estonian bryophytes. Folia Cryptogamica Estonica 55: 97-104. https://doi. org/10.12697/fce.2018.55.10

IUCN Standards and Petitions Subcommittee, 2017. Guidelines for Using the IUCN Red List Categories and Criteria. Version 13. Prepared by the Standards and Petitions Subcommittee. http://www.iucnredlist.org/documents / RedListGuidelines.pdf.

Jüriado, I. \& Liira, J. 2009. Distribution and habitat ecology of the threatened forest lichen Lobaria pulmonaria in Estonia. Folia Cryptogamica Estonica 46: 55-65.

Jüriado, I. \& Liira, J. 2010. Threatened forest lichen Lobaria pulmonaria-its past, present and future in Estonia. Metsanduslikud Uurimused (Forestry Studies) 53: 15-24. https://doi.org/10.2478/ v10132-011-0086-6
Jüriado, I. \& Paal, J. 2019. Epiphytic lichen synusiae and functional trait groups in boreo-nemoral deciduous forests are influenced by host tree and environmental factors. Nordic Journal of Botany 37: e01939. https://doi.org/10.1111/njb.01939

Jüriado, I., Kämärä, M.-L. \& Oja, E. 2016. Environmental factors and ground disturbance affecting the composition of species and functional traits of ground layer lichens on grey dunes and dune heaths of Estonia. Nordic Journal of Botany 34(2): 244-255. https://doi.org/10.1111/njb.00936

Jüriado, I., Paal, J. \& Liira, J. 2003. Epiphytic and epixylic lichen species diversity in Estonian natural forests. Biodiversity and Conservation 12: 1587-1607. https://doi.org/10.1023/A: 1023645730446

Keskkonnaministri 19. mai 2004. a. määrus nr 51. III kaitsekategooria liikide kaitse alla võtmine. (Decree of the Estonian Minister of Environment no 51. 19.05.2004) Riigi Teataja Lisa 27.05.2004, 69, 1134. (In Estonian).

Leppik, E., Jüriado, I., Suija, A. \& Liira, J. 2013. The conservation of ground layer lichen communities in alvar grasslands and the relevance of substitution habitats. Biodiversity and Conservation 22: 591-614. https://doi.org/10.1007/s10531-0120430-z

Leppik, E., Jüriado, I., Suija, A. \& Liira, J. 2015. Functional ecology of rare and common epigeic lichens in alvar grasslands. Fungal Ecology 13: 66-76. https://doi.org/10.1016/j. funeco.2014.08.003

Lõhmus, P. \& Kruustük, K. 2010. Lichens on burnt wood in Estonia: a preliminary assessment. Folia Cryptogamica Estonica 47: 37-41.

Lõhmus, A. \& Lõhmus, P. 2011. Old-forest species: the importance of specific substrata vs. stand continuity in the case of calicioid fungi. Silva Fennica 45(5): 1015-1039. https://doi. org/ 10.14214/sf.84

Lõhmus, A., Lõhmus, P. \& Runnel, K. 2018. A simple survey protocol for assessing terrestrial biodiversity in a broad range of ecosystems. PloS One 13(12), e0208535. https://doi.org/10.1371/ journal.pone.0208535

Mark, K., Saag, L., Leavitt, S. D., Will-Wolf, S., Nelsen, M. P., Törra, T., Saag, A., Randlane, T. \& Lumbsch, H. T. 2016. Evaluation of traditionally circumscribed species in the lichen-forming genus Usnea, section Usnea (Parmeliaceae, Ascomycota) using a six-locus dataset. Organisms Diversity \& Evolution 16: 497-524. https://doi.org/10.1007/ s13127-016-0273-7

Marmor, L., Randlane, T., Jüriado, I. \& Saag, A. 2017. Host tree preferences of red-listed epiphytic lichens in Estonia. Baltic Forestry 23(2): 364-373.

Paal, J. 1997. Classification of Estonian vegetation site types. Eesti Keskkonnaministeerium \& ÜRO Keskonnaprogramm, Tallinn. 297 p. (In Estonian). 
Randlane, T., Saag, A. \& Suija, A. 2006. Lichenized, lichenicolous and allied fungi of Estonia. Ver. April 24, 2006. http://eseis.ut.ee/ial5/lich/e_liigid/ samblik_e_2006.html

Randlane, T., Saag, A. \& Suija, A. 2018. Lichenized, lichenicolous and allied fungi of Estonia. Ver. December 31, 2018. http://esamba.bo.bg.ut-ee/ checklist/est/home.php

Randlane, T., Jüriado, I., Suija, A., Lõhmus, P. \& Leppik, E. 2008. Lichens in the new Red List of Estonia. Folia Cryptogamica Estonica 44: 113-120.

Saag, L., Mark, K., Saag, A. \& Randlane, T. 2014. Species delimitation in the lichenized fungal genus Vulpicida (Parmeliaceae, Ascomycota) using gene concatenation and coalescent-based species tree approaches. American Journal of Botany 101(12): 2169-2182. https://doi.org/10.3732/ ajb. 1400439
Standards and Petitions Working Group 2006. Guidelines for Using the IUCN Red List Categories and Criteria: Version 6.1. Prepared by the Standards and Petitions Working Group for the IUCN SSC Biodiversity Assessments SubCommittee in July 2006. Downloadable from http://app.iucn.org/webfiles/doc/SSC/RedList/ RedListGuidelines.pdf.

Tartes, U. 2010. Punane nimestik ja liigikaitse. In: Eesti Teaduste Akadeemia seminari materjalid. Rahvusvahelise elurikkuse aasta 2010. Tallinn, 1k. 4-8. (In Estonian).

Vabariigi Valitsuse 20. mai 2004. a määrus nr 195. I ja II kaitsekategooriana kaitse alla võetavate liikide loetelu. (Decree of the Estonian Government no 195. 20.05.2004) Riigi Teataja I 21.05.2004, 44, 313. (In Estonian). 
Supplement 1. Red-listed lichens according to the evaluation in 2019 together with the IUCN categories and applied criteria; details of the previous assessment (incl. species older names used in Table 2, Randlane et al., 2008) are also given. For abbreviations of categories and criteria see Material and Methods. Comment field provides information about the protection category (I-III) and the year of the last documented record for the species that were assessed as RE or being very close to it. The taxonomy of the species follows Randlane et al. (2018).

\begin{tabular}{|c|c|c|c|c|c|c|}
\hline Species name & $\begin{array}{l}\text { Species } \\
\text { name used } \\
\text { in } 2008\end{array}$ & $\begin{array}{l}\text { Cat- } \\
\text { egory } \\
2019\end{array}$ & Criteria 2019 & $\begin{array}{l}\text { Cat- } \\
\text { egory } \\
2008\end{array}$ & Criteria 2008 & $\begin{array}{l}\text { Com- } \\
\text { ment }\end{array}$ \\
\hline Acarospora oligospora (Nyl.) Arnold & & RE & & RE & & $<1890$ \\
\hline Alectoria sarmentosa (Ach.) Ach. & & VU & $\mathrm{C} 1 ; \mathrm{D} 1$ & NT & & II \\
\hline Alyxoria culmigena (Lib.) Ertz & $\begin{array}{l}\text { Opegrapha } \\
\text { herbarum }\end{array}$ & EN & $\mathrm{D}$ & $\mathrm{DD}$ & & \\
\hline Alyxoria ochrocheila (Nyl.) Ertz \& Tehler & $\begin{array}{l}\text { Opegrapha } \\
\text { ochrocheila }\end{array}$ & NT & A2c; B2ab(iii) & VU & D2 & \\
\hline Amygdalaria panaeola (Ach.) Hertel \& Brodo & & $\mathrm{RE}$ & & $\mathrm{RE}$ & & $<1890$ \\
\hline Anaptychia runcinata (With.) J. R. Laundon & & CR & $\mathrm{D}$ & VU & D2 & \\
\hline Arctoparmelia centrifuga (L.) Hale & & VU & $\begin{array}{l}\text { A2c; } \\
\text { B1ab(i,iii,iv) }\end{array}$ & EN & B1ab(i,iv) & \\
\hline Arctoparmelia incurva (Pers.) Hale & & EN & $\begin{array}{l}\text { A2c; } \\
\text { B2ab(ii,iii,iv) }\end{array}$ & EN & A2c; B1ab(i,iv) & \\
\hline Arthonia apatetica (A. Massal.) Th. Fr. & & VU & D1 & VU & D2 & \\
\hline Arthonia atra (Pers.) A. Schneid. & $\begin{array}{l}\text { Opegrapha } \\
\text { atra }\end{array}$ & VU & $\mathrm{C} 1+2 \mathrm{a}(\mathrm{i}) ; \mathrm{D} 1$ & NT & & \\
\hline Arthonia byssacea (Weigel) Almq. & & VU & $\mathrm{C} 1 ; \mathrm{D} 1$ & NT & & III \\
\hline Arthonia didyma Körb. & & NT & $\mathrm{A} 2 \mathrm{c}+3 \mathrm{c} ; \mathrm{C} 1$ & NT & & \\
\hline Arthonia lapidicola (Taylor) Branth. \& Rostr. & & VU & D1 & VU & D2 & \\
\hline Arthopyrenia cinereopruinosa (Schaer.) A. Massal. & & $\mathrm{RE}$ & & $\mathrm{RE}$ & & $<1890$ \\
\hline Arthothelium spectabile Flot. ex A. Massal. & & $\mathrm{CR}$ & $\mathrm{D}$ & VU & D2 & \\
\hline Aspicilia zonata (Ach.) R. Sant. & $\begin{array}{l}\text { Aspicilia } \\
\text { xyloxena }\end{array}$ & $\mathrm{RE}$ & & $\mathrm{RE}$ & & 1945 \\
\hline Bacidia biatorina (Körb.) Vain. & & VU & $\begin{array}{l}\text { B1ab(iii) }+2 a b(\text { iii); } \\
\text { C1; D1 }\end{array}$ & EN & B2ab(iii) & II \\
\hline Bacidia fuscoviridis (Anzi) Lettau & & $\mathrm{RE}$ & & $\mathrm{RE}$ & & 1929 \\
\hline Bacidia laurocerasi (Delise ex Duby) Zahlbr. & & VU & C1; D1 & NT & & II \\
\hline Baeomyces carneus Flörke & & $\mathrm{EN}$ & $\mathrm{C} 2 \mathrm{a}(\mathrm{i}) ; \mathrm{D}$ & VU & D2 & II \\
\hline Biatoridium monasteriense J. Lahm ex Körb. & & VU & $\mathrm{A} 2 \mathrm{c}$ & NT & & II \\
\hline $\begin{array}{l}\text { Bilimbia lobulata (Sommerf.) Hafellner \& } \\
\text { Coppins }\end{array}$ & & EN & $\mathrm{C} 2 \mathrm{a}(\mathrm{i}) ; \mathrm{D}$ & VU & B2ab(iii) & \\
\hline Bryoria chalybeiformis (L.) Brodo \& D. Hawksw. & & EN & $\mathrm{D}$ & VU & D2 & \\
\hline Bryoria furcellata (Fr.) Brodo \& D. Hawksw. & & EN & $\mathrm{D}$ & VU & B1ab(iii) & II \\
\hline Bryoria simplicior (Vain.) Brodo \& D. Hawksw. & & $\mathrm{CR}$ & $\mathrm{D}$ & DD & & 1963 \\
\hline $\begin{array}{l}\text { Calogaya biatorina (A. Massal.) Arup, Frödén \& } \\
\text { Søchting }\end{array}$ & $\begin{array}{l}\text { Caloplaca } \\
\text { biatorina }\end{array}$ & EN & $\mathrm{D}$ & VU & D2 & \\
\hline Caloplaca atroflava (Turner) Mong. & & $\mathrm{RE}$ & & $\mathrm{RE}$ & & $<1890$ \\
\hline Caloplaca chalybaea (Fr.) Müll. Arg. & & $\mathrm{RE}$ & & $\mathrm{RE}$ & & 1908 \\
\hline Caloplaca lucifuga $\mathrm{G}$. Thor & & EN & $\mathrm{D}$ & NT & & \\
\hline Caloplaca ulcerosa Coppins \& P. James & & VU & D1 & VU & D2 & \\
\hline $\begin{array}{l}\text { Carbonicola anthracophila (Nyl.) Bendiksby \& } \\
\text { Timdal }\end{array}$ & $\begin{array}{l}\text { Hypoceno- } \\
\text { myce an- } \\
\text { thracophila }\end{array}$ & VU & $\mathrm{A} 3 \mathrm{C}$ & $\mathrm{LC}$ & & II \\
\hline
\end{tabular}




Species name

in

$\begin{array}{llllll}\begin{array}{l}\text { Species } \\ \text { name used } \\ \text { in 2008 }\end{array} & \begin{array}{l}\text { Cat- } \\ \text { egory } \\ 2019\end{array} & \text { Criteria 2019 } & \begin{array}{l}\text { Cat- } \\ \text { egory } \\ 2008\end{array} & \text { Criteria 2008 } & \begin{array}{l}\text { Com- } \\ \text { ment }\end{array} \\ & \text { CR } & \text { C2a(i); D } & - & & \\ & \text { EN } & \begin{array}{l}\text { B1ab(iii)+2ab(iii); EN } \\ \text { D }\end{array} & \text { B2ab(iii) } & \\ \text { EN } & \text { C1+C2a(i) } & \text { VU } & \text { B1ab(iii) } & \\ & \text { CR } & \text { C2a(i) } & - & & \\ & \text { EN } & \text { B2ab(iii); } & \text { VU } & \text { B1ab(iii) } & \text { II } \\ \text { CR } & \text { C22a(i) } & \text { EN } & \text { B2ab(iii) } & \text { II } \\ \text { EN } & \text { C2a(i) } & \text { VU } & \text { B2ab(iii) } & \text { II }\end{array}$
Middelb.

Circinaria gibbosa (Ach.) A. Nordin, Savić \& Tibell

Cladonia borealis S. Stenroos

Aspicilia
gibbosa

Cladonia brevis (Sandst.) Sandst.

Cladonia caespiticia (Pers.) Flörke

Cladonia carneola (Fr.) Vain.

Cladonia cervicornis (Ach.) Flot.

Cladonia coccifera (L.) Willd.

Cladonia cryptochlorophaea Asahina

Cladonia decorticata (Flörke) Spreng.

Cladonia foliacea (Huds.) Willd.

Cladonia grayi G. Merr. ex Sandst.

Cladonia humilis (With.) J.R. Laundon

Cladonia incrassata Flörke

Cladonia macroceras (Delise) Hav.

Cladonia macrophylla (Schaer.) Stenh.

Cladonia magyarica Vain. ex Gyeln.

Cladonia merochlorophaea Asahina

Cladonia novochlorophaea (Sipman) Brodo \& Ahti

Cladonia parasitica (Hoffm.) Hoffm.

Cladonia pocillum (Ach.) Grognot

Cladonia portentosa (Dufour) Follmann

Cladonia ramulosa (With.) J.R. Laundon

Cladonia scabriuscula (Delise) Nyl.

Cladonia straminea (Sommerf.) Flörke

Coenogonium luteum (Dicks.) Kalb \& Lücking

Collema flaccidum (Ach.) Ach.

RE

RE

1931

VU

$\mathrm{A} 2 \mathrm{c} ; \mathrm{C} 1+2 \mathrm{a}(\mathrm{i})$;

EN A2bc

CR D

EN B2ab(iii)

1958

CR D

VU D2

VU A2c; C1; D1

EN A2bc

EN C2a(i); D

DD

EN

D

EN A2bc

DD

NE

EN B2ab(iii); D VU

D2

NT A2c+4c; D NT

NT D NE

VU C1; D1

VU D1 NT

VU A2c; $\mathrm{C} 1+\mathrm{C} 2 \mathrm{a}(\mathrm{i}) ; \quad \mathrm{NT}$

D1

EN A2c;

B2ab(i,ii,iii);

$\mathrm{C} 1+2 \mathrm{a}(\mathrm{i}) ; \mathrm{D}$

EN A2bc;

A2bc;
B2ab(iii)

VU

B2ab(iii); D1

DD

NE

VU B2ab(iii); C1; NE

D1

VU C1

NT

NT C1

NT

NT D

NT

EN D

VU D1

Cladonia

metacoral-

CR D

VU A2bc

VU D2

lifera

Dimerella
lutea

EN

A2c; C2a(i); D

VU

B2ab(iii)

II

EN D

NE 


\begin{tabular}{|c|c|c|c|c|c|c|}
\hline Species name & $\begin{array}{l}\text { Species } \\
\text { name used } \\
\text { in } 2008\end{array}$ & $\begin{array}{l}\text { Cat- } \\
\text { egory } \\
2019 \\
\end{array}$ & Criteria 2019 & $\begin{array}{l}\text { Cat- } \\
\text { egory } \\
2008 \\
\end{array}$ & Criteria 2008 & $\begin{array}{l}\text { Com- } \\
\text { ment }\end{array}$ \\
\hline Collema nigrescens (Huds.) DC. & & EN & $\mathrm{C} 2 \mathrm{a}(\mathrm{i})$ & VU & B2ab(iii); C1 & II \\
\hline Collema subnigrescens Degel. & & EN & $\mathrm{C} 2 \mathrm{a}(\mathrm{i})$ & NT & & \\
\hline Cyphelium inquinans (Sm.) Trevis. & & $\mathrm{EN}$ & $\mathrm{D}$ & NT & & III \\
\hline Dermatocarpon luridum (With.) J. R. Laundon & & CR & $\mathrm{B} 2 \mathrm{ab}(\mathrm{i}, \mathrm{ii}) ; \mathrm{C} 2 \mathrm{a}(\mathrm{i})$ & DD & & \\
\hline Dermatocarpon miniatum (L.) W. Mann & & EN & $\mathrm{D}$ & $\mathrm{DD}$ & & \\
\hline Dibaeis baeomyces (L. f.) Rambold \& Hertel & & EN & $\mathrm{C} 2 \mathrm{a}(\mathrm{i}) ; \mathrm{D}$ & NT & & \\
\hline Diplotomma lutosum (Ach.) Arnold & & $\mathrm{CR}$ & $\mathrm{D}$ & $\mathrm{RE}$ & & \\
\hline $\begin{array}{l}\text { Enchylium bachmanianum (Fink) Otálora, } \\
\text { P.M. Jørg. \& Wedin }\end{array}$ & $\begin{array}{l}\text { Collema } \\
\text { bachmani- } \\
\text { anum }\end{array}$ & $\mathrm{EN}$ & $\mathrm{D}$ & DD & & \\
\hline $\begin{array}{l}\text { Enchylium limosum (Ach.) Otálora, P.M. Jørg. \& } \\
\text { Wedin }\end{array}$ & $\begin{array}{l}\text { Collema } \\
\text { limosum }\end{array}$ & VU & B2ab(iii); D1 & $\mathrm{DD}$ & & \\
\hline Enchylium tenax (Sw.) Gray & & NT & $\mathrm{A} 2 \mathrm{c}+4 \mathrm{c} ; \mathrm{D}$ & NE & & \\
\hline Endocarpon psorodeum (Nyl.) Blomb. \& Forssell & & $\mathrm{EN}$ & $\mathrm{D}$ & EN & $\mathrm{D}$ & \\
\hline Endocarpon pusillum Hedw. & & $\mathrm{EN}$ & $\mathrm{C} 2 \mathrm{a}(\mathrm{i}) ; \mathrm{D}$ & $\mathrm{EN}$ & B2ab(iii) & \\
\hline Eopyrenula leucoplaca (Wallr.) R. C. Harris & & $\mathrm{EN}$ & $\mathrm{D}$ & EN & B2ab(iii) & \\
\hline Evernia divaricata (L.) Ach. & & VU & $\mathrm{A} 2 \mathrm{c} ; \mathrm{C} 1$ & VU & A4bc & III \\
\hline Evernia mesomorpha $\mathrm{Nyl}$. & & VU & $\mathrm{C} 1 ; \mathrm{D} 1$ & NT & & \\
\hline $\begin{array}{l}\text { Flavocetraria cucullata (Bellardi) Kärnefelt \& } \\
\text { A. Thell }\end{array}$ & & $\mathrm{CR}$ & $\begin{array}{l}\text { A2a; } \\
\text { B1ab(i,iii,iii)+ } \\
\text { 2ab(i,ii,iii) }\end{array}$ & $\mathrm{CR}$ & $\begin{array}{l}\mathrm{B} 1 \mathrm{ab}(\mathrm{iii})+ \\
2 \mathrm{ab}(\mathrm{iii})\end{array}$ & I \\
\hline Flavocetraria nivalis (L.) Kärnefelt \& A. Thell & & CR & A2ac & NT & & \\
\hline Flavoparmelia caperata (L.) Hale & & EN & $\mathrm{D}$ & $\mathrm{EN}$ & B1ab(iii) & II \\
\hline Fuscidea cyathoides (Ach.) V. Wirth \& Vězda & & $\mathrm{RE}$ & & $\mathrm{RE}$ & & $<1890$ \\
\hline Gyalecta ulmi (Sw.) Zahlbr. & & EN & $\mathrm{A} 2 \mathrm{c} ; \mathrm{C} 1+2 \mathrm{a}(\mathrm{i}) ; \mathrm{D}$ & VU & B2ab(iii) & II \\
\hline Gyalolechia bracteata (Hoffm.) A. Massal. & $\begin{array}{l}\text { Fulgensia } \\
\text { bracteata }\end{array}$ & NT & $\mathrm{A} 2 \mathrm{c}+4 \mathrm{c} ; \mathrm{C} 1$ & NT & & III \\
\hline Gyalolechia fulgens (Sw.) Søchting, Frödén \& Arup & $\begin{array}{l}\text { Fulgensia } \\
\text { fulgens }\end{array}$ & CR & $\mathrm{D}$ & $\mathrm{DD}$ & & \\
\hline Hypogymnia vittata (Ach.) Parrique & & $\mathrm{CR}$ & $\mathrm{D}$ & $\mathrm{CR}$ & B2ab(iii) & 1965 \\
\hline Lasallia pustulata (L.) Mérat & & $\mathrm{EN}$ & $\mathrm{A} 2 \mathrm{c} ; \mathrm{D}$ & VU & $\mathrm{A} 2 \mathrm{c}+3 \mathrm{c}$ & III \\
\hline $\begin{array}{l}\text { Lathagrium cristatum (L.) Otálora, P.M. Jørg. \& } \\
\text { Wedin }\end{array}$ & & VU & D1 & NE & & \\
\hline $\begin{array}{l}\text { Lathagrium undulatum (Flot.) Otálora, P.M. Jørg. } \\
\text { \& Wedin }\end{array}$ & $\begin{array}{l}\text { Collema } \\
\text { undulatum }\end{array}$ & CR & $\mathrm{D}$ & VU & D2 & \\
\hline Lecanora bicincta Ramond & & $\mathrm{CR}$ & $\mathrm{D}$ & VU & $\mathrm{D} 2$ & \\
\hline Lecanora caesiosora Poelt & & CR & B1ab(iii); D & VU & D2 & \\
\hline Lecanora epibryon (Ach.) Ach. & & CR & $\begin{array}{l}\text { B1ab(iii) }+2 a b(\text { iii }) \text {; } \\
\text { D }\end{array}$ & $\mathrm{RE}$ & & \\
\hline Lecanora impudens Degel. & & $\mathrm{EN}$ & B2ab(iii); D & VU & D2 & \\
\hline Lecanora intumescens (Rebent.) Rabenh. & & EN & $\mathrm{C} 2 \mathrm{a}(\mathrm{i})$ & VU & B2ab(iii) & \\
\hline Lecidea erythrophaea Flörke ex Sommerf. & & NT & $\mathrm{A} 3 \mathrm{c}$ & NT & & III \\
\hline $\begin{array}{l}\text { Lempholemma isidiodes (Nyl. ex Arnold) } \\
\text { H. Magn. }\end{array}$ & & CR & $\mathrm{D}$ & VU & D2 & \\
\hline Lempholemma polyanthes (Bernh.) Malme & & $\mathrm{RE}$ & & $\mathrm{RE}$ & & 1929 \\
\hline Leptogium rivulare (Ach.) Mont. & & $\mathrm{CR}$ & $\mathrm{D}$ & CR & $\mathrm{D}$ & 1957 \\
\hline
\end{tabular}




Species name

Species name

in

Leptogium saturninum (Dicks.) Nyl.

Lobaria pulmonaria (L.) Hoffm.

Lobaria scrobiculata (Scop.) DC.

Lobothallia radiosa (Hoffm.) Hafellner

Megalaria grossa (Pers. ex Nyl.) Hafellner

Megaspora verrucosa (Ach.) Hafellner \& V. Wirth

Melanelia commixta (Nyl.) A. Thell

Melanelia disjuncta (Erichsen) Essl.

Melanelia hepatizon (Ach.) A. Thell

Melanelia panniformis (Nyl.) Essl.

Melanelia sorediata (Ach.) Goward \& Ahti

Melanelia stygia (L.) Essl.

Melanelixia glabra (Schaer.) O. Blanco, A. Crespo, Melanelia Divakar, Essl., D. Hawksw. \& Lumbsch

Melanelixia glabratula (Lamy) Sandler \& Arup

Melanohalea elegantula (Zahlbr.) O. Blanco, A. Crespo, Divakar, Essl., D. Hawksw. \& Lumbsch

Melanohalea septentrionalis (Lynge) O. Blanco, A. Crespo, Divakar, Essl., D. Hawksw. \& Lumbsch

Melaspilea gibberulosa (Ach.) Zwackh

Menegazzia terebrata (Hoffm.) A. Massal.

Micarea hedlundii Coppins

Micarea turfosa (A. Massal.) Du Rietz

Multiclavula mucida (Pers.) R. H. Petersen

Multiclavula vernalis (Schwein.) R. H. Petersen

Nephroma arcticum (L.) Torss.

Nephroma bellum (Spreng.) Tuck.

Nephroma isidiosum (Nyl.) Gyeln.

Nephroma laevigatum Ach.

Nephroma parile (Ach.) Ach.

Nephroma resupinatum (L.) Ach.

Ochrolechia frigida (Sw.) Lynge

Parmelia fraudans (Nyl.) Nyl.

Parmelia omphalodes (L.) Ach.

Parmelia submontana Nádv. ex Hale

Parmeliella triptophylla (Ach.) Müll. Arg.

Parmelina tiliacea (Hoffm.) Hale

\begin{tabular}{|c|c|c|c|c|c|}
\hline $\begin{array}{l}\text { Species } \\
\text { name used } \\
\text { in } 2008\end{array}$ & $\begin{array}{l}\text { Cat- } \\
\text { egory } \\
2019 \\
\end{array}$ & Criteria 2019 & $\begin{array}{l}\text { Cat- } \\
\text { egory } \\
2008 \\
\end{array}$ & Criteria 2008 & $\begin{array}{l}\text { Com- } \\
\text { ment }\end{array}$ \\
\hline & $\mathrm{VU}$ & $\mathrm{A} 2 \mathrm{c}+3 \mathrm{c} ; \mathrm{C} 1$ & NT & & III \\
\hline & VU & $\begin{array}{l}\mathrm{A} 2 \mathrm{bc}+4 \mathrm{bc} \\
\mathrm{C} 1+2 \mathrm{a}(\mathrm{i})\end{array}$ & NT & & III \\
\hline & CR & B2ab(iii); D & CR & B2ab(iii) & \\
\hline & $\mathrm{EN}$ & $\begin{array}{l}\text { B1ab(iii)+2ab(iii); } \\
\text { D }\end{array}$ & NT & & \\
\hline & $\mathrm{VU}$ & $\mathrm{C} 1$ & NT & & III \\
\hline & CR & $\begin{array}{l}\text { B1ab(iii)+2ab(iii); } \\
\text { C2a(i); D }\end{array}$ & EN & B2ab(iii) & \\
\hline & CR & $\mathrm{D}$ & CR & $\begin{array}{l}\text { B2ab(iv); } \\
\text { C1+2a(i) }\end{array}$ & \\
\hline & $\mathrm{EN}$ & $\mathrm{A} 2 \mathrm{c}$ & DD & & \\
\hline & EN & $\mathrm{D}$ & EN & B1ab(iii,iv) & \\
\hline & CR & $\mathrm{D}$ & - & & \\
\hline & $\mathrm{EN}$ & $\mathrm{A} 2 \mathrm{c} ; \mathrm{D}$ & DD & & \\
\hline & $\mathrm{EN}$ & $\mathrm{D}$ & VU & $\mathrm{A} 2 \mathrm{c}$ & \\
\hline \multirow[t]{2}{*}{$\begin{array}{l}\text { Melanelia } \\
\text { glabra }\end{array}$} & CR & $\begin{array}{l}\text { B1ab(iii)+2ab(iii); } \\
\text { D }\end{array}$ & CR & B2ab(iii) & 1958 \\
\hline & NT & $\mathrm{A} 2 \mathrm{c}$ & - & & \\
\hline $\begin{array}{l}\text { Melanelia } \\
\text { elegantula }\end{array}$ & CR & $\begin{array}{l}\text { B1ab(iii)+2ab(iii); } \\
\text { D }\end{array}$ & EN & B1ab(iii,iv) & \\
\hline \multirow{19}{*}{$\begin{array}{l}\text { Melanelia } \\
\text { septentri- } \\
\text { onalis }\end{array}$} & $\mathrm{EN}$ & $\mathrm{D}$ & NT & & \\
\hline & $\mathrm{RE}$ & & $\mathrm{RE}$ & & 1929 \\
\hline & VU & $\mathrm{C} 1+2 \mathrm{a}(\mathrm{i}) ; \mathrm{D} 1$ & NT & & III \\
\hline & VU & $A 2 c+3 c$ & VU & B2ab(iii) & II \\
\hline & $\mathrm{RE}$ & & $\mathrm{RE}$ & & 1929 \\
\hline & VU & $\mathrm{A} 2 \mathrm{c} ; \mathrm{C} 1 ; \mathrm{D} 1$ & VU & B2ab(iii) & \\
\hline & $\mathrm{EN}$ & $\mathrm{D}$ & CR & A2a; D & \\
\hline & $\mathrm{RE}$ & & $\mathrm{RE}$ & & 1882 \\
\hline & CR & $\begin{array}{l}\text { B1ab(iii)+2ab(iii); } \\
\text { D }\end{array}$ & CR & B2ab(iii) & \\
\hline & CR & $\begin{array}{l}\text { B1ab(iii)+2ab(iii); } \\
\text { D }\end{array}$ & DD & & 1961 \\
\hline & $\mathrm{EN}$ & $\mathrm{C} 1+\mathrm{C} 2 \mathrm{a}(\mathrm{i})$ & VU & A $4 b c$ & III \\
\hline & $\mathrm{EN}$ & $\mathrm{A} 2 \mathrm{bc} ; \mathrm{C} 1+\mathrm{C} 2 \mathrm{a}(\mathrm{i})$ & $\mathrm{VU}$ & $\mathrm{A} 2 \mathrm{bc}$ & III \\
\hline & CR & C2a(i) & EN & A2bc & II \\
\hline & VU & D1 & VU & B1ab(iii) & II \\
\hline & CR & $\begin{array}{l}\text { B1ab(iii)+2ab(iii); } \\
\text { D }\end{array}$ & EN & B1ab(i,iv) & \\
\hline & VU & $\mathrm{A} 2 \mathrm{c} ; \mathrm{C} 1 ; \mathrm{D} 1$ & NT & & \\
\hline & CR & $\mathrm{D}$ & - & & \\
\hline & VU & $\mathrm{C} 1+2 \mathrm{a}(\mathrm{i}) ; \mathrm{D} 1$ & VU & $\begin{array}{l}\text { A2bc; } \\
\text { B2ab(iii) }\end{array}$ & II \\
\hline & VU & $\mathrm{C} 1: \mathrm{D} 1$ & $\mathrm{NT}$ & & \\
\hline
\end{tabular}




\begin{tabular}{|c|c|c|c|c|c|c|}
\hline Species name & $\begin{array}{l}\text { Species } \\
\text { name used } \\
\text { in } 2008\end{array}$ & $\begin{array}{l}\text { Cat- } \\
\text { egory } \\
2019 \\
\end{array}$ & Criteria 2019 & $\begin{array}{l}\text { Cat- } \\
\text { egory } \\
2008 \\
\end{array}$ & Criteria 2008 & $\begin{array}{l}\text { Com- } \\
\text { ment }\end{array}$ \\
\hline $\begin{array}{l}\text { Peltigera castanea Goward, Goffinet } \& \\
\text { Miadlikowska }\end{array}$ & & CR & $\begin{array}{l}\text { B1ab(iii)+2ab(iii); } \\
\text { D }\end{array}$ & - & & \\
\hline Peltigera collina (Ach.) Schrad. & & CR & $\mathrm{C} 2 \mathrm{a}(\mathrm{i})$ & CR & $\mathrm{D}$ & II \\
\hline Peltigera degenii Gyeln. & & EN & B2ab(iii); D & VU & D2 & \\
\hline Peltigera elisabethae Gyeln. & & CR & C2a(i); D & $\mathrm{RE}$ & & \\
\hline Peltigera horizontalis (Huds.) Baumg. & & EN & & NT & & \\
\hline Peltigera hymenina (Ach.) Delise & & NT & B2ab(iii) & NT & & \\
\hline Peltigera islandica Goward \& Manoharan-Basil & & CR & $\mathrm{D}$ & - & & \\
\hline Peltigera lepidophora (Nyl. ex Vain.) Bitter & & VU & $\begin{array}{l}\text { A2bc; B2ab(iii); } \\
\text { C1+2a(i) }\end{array}$ & $\mathrm{DD}$ & & \\
\hline Peltigera occidentalis (E. Dahl) Kristinsson & & CR & $\begin{array}{l}\text { B1ab(iii)+2ab(iii); } \\
\text { D }\end{array}$ & - & & \\
\hline Peltigera ponojensis Gyeln. & & NT & $\begin{array}{l}\text { B1ab(iii) }+2 \mathrm{ab}(\mathrm{iii}) \\
\text { C1 }\end{array}$ & $\mathrm{DD}$ & & \\
\hline Peltigera scabrosa Th. Fr. & & CR & $\mathrm{D}$ & EN & B2ab(iii) & \\
\hline Peltigera venosa (L.) Hoffm. & & CR & $\mathrm{D}$ & $\mathrm{EN}$ & B2ab(iv) & 1961 \\
\hline Pertusaria carneopallida (Nyl.) Anzi & & $\mathrm{RE}$ & & $\mathrm{RE}$ & & 1946 \\
\hline Phaeophyscia chloantha (Ach.) Moberg & & $\mathrm{RE}$ & & $\mathrm{RE}$ & & 1927 \\
\hline Phaeophyscia endophoenicea (Harm.) Moberg & & EN & B2ab(iii); D & $\mathrm{DD}$ & & \\
\hline Physcia leptalea (Ach.) DC. & & EN & $\mathrm{D}$ & VU & A4c & \\
\hline Physcia magnussonii Frey & & CR & $\mathrm{D}$ & VU & $\mathrm{D} 2$ & \\
\hline Physconia detersa (Nyl.) Poelt & & VU & D1 & NT & & \\
\hline Physconia grisea (Lam.) Poelt & & EN & A2c; B2ab(iii) & NT & & \\
\hline Pilophorus cereolus (Ach.) Th. Fr. & & CR & $\mathrm{D}$ & EN & B2ab(iii) & 1969 \\
\hline Placidium pilosellum (Breuss) Breuss & & EN & $\mathrm{C} 2 \mathrm{a}(\mathrm{i})$ & $\mathrm{EN}$ & B2ab(iii) & \\
\hline Placidium squamulosum (Ach.) Breuss & & NT & $\mathrm{A} 2 \mathrm{c}+3 \mathrm{c} ; \mathrm{C} 1$ & $\mathrm{EN}$ & B2ab(iii) & \\
\hline $\begin{array}{l}\text { Polycauliona verruculifera (Vain.) Arup, Frödén \& } \\
\text { Søchting }\end{array}$ & $\begin{array}{l}\text { Caloplaca } \\
\text { verrucu- } \\
\text { lifera }\end{array}$ & EN & $\mathrm{D}$ & NT & & II \\
\hline Polychidium muscicola (Sw.) Gray & & EN & $\mathrm{D}$ & DD & & \\
\hline $\begin{array}{l}\text { Protopannaria pezizoides (Weber) P. M. Jørg. \& } \\
\text { S. Ekman }\end{array}$ & & CR & $\mathrm{D}$ & DD & & 1965 \\
\hline $\begin{array}{l}\text { Protoparmeliopsis achariana (A. L. Sm.) Moberg } \\
\quad \text { \& R. Sant. }\end{array}$ & & CR & $\mathrm{D}$ & VU & D2 & 1964 \\
\hline $\begin{array}{l}\text { Protoparmeliopsis macrocyclos (H. Magn.) Moberg } \\
\quad \text { \& R. Sant. }\end{array}$ & & NT & B1ab(iii) & NT & & \\
\hline Psora decipiens (Hedw.) Hoffm. & & NT & $\mathrm{A} 2 \mathrm{c}+3 \mathrm{c} ; \mathrm{C} 1$ & NT & & III \\
\hline Punctelia subrudecta (Nyl.) Krog & & $\mathrm{RE}$ & & $\mathrm{RE}$ & & 1947 \\
\hline Pycnora praestabilis (Nyl.) Hafellner & & CR & A2a; B2ab(iii); D & CR & $\begin{array}{l}\text { B2ab(i,ii,iii,iv); } \\
\text { D }\end{array}$ & \\
\hline Pycnothelia papillaria Dufour & & EN & B2ab(iii) & EN & A2 & \\
\hline Pyrenula laevigata (Pers.) Arnold & & EN & $\mathrm{C} 1+2 \mathrm{a}(\mathrm{i}) ; \mathrm{D}$ & VU & B2ab(iii) & II \\
\hline Pyrenula nitidella (Schaer.) Müll. Arg. & & $\mathrm{EN}$ & $\mathrm{C} 1+2 \mathrm{a}(\mathrm{i}) ; \mathrm{D}$ & VU & B2ab(iii) & II \\
\hline Ramalina calicaris (L.) Fr. & & EN & $\mathrm{D}$ & VU & A1b & \\
\hline Ramalina dilacerata (Hoffm.) Hoffm. & & CR & $\mathrm{D}$ & DD & & \\
\hline Ramalina elegans (Bagl. \& Carestia) Jatta & & $\mathrm{DD}$ & & $\mathrm{RE}$ & & \\
\hline
\end{tabular}




Species name

Ramalina obtusata (Arnold) Bitter

Ramalina siliquosa (Huds.) A. L. Sm.

Ramalina sinensis Jatta

Ramalina thrausta (Ach.) Nyl.

Rhizocarpon badioatrum (Flörke ex Spreng.)

$$
\text { Th. Fr. }
$$

Rhizocarpon oederi (Weber) Körb.

Rinodina interpolata (Stirt.) Sheard

Rostania occultata (Bagl.) Otálora, P.M. Jørg. \& Wedin

Rusavskia sorediata (Vain.) S.Y. Kondr. \& Kärnefelt

Sclerophora coniophaea (Norman) J. Mattsson \& Middelb.

Sclerophora farinacea (Chevall.) Chevall.

Sclerophora pallida (Pers.) Y. I. Yao \& Spooner

Sclerophora peronella (Ach.) Tibell

Scytinium aragonii (Otálora) Otálora, P.M. Jørg. \& Wedin

Scytinium gelatinosum (With.) Otálora, P.M. Jørg. \& Wedin

Scytinium imbricatum (P.M. Jørg.) Otálora,

P.M. Jørg. \& Wedin

Scytinium intermedium (Arnold) Otálora,

P.M. Jørg. \& Wedin

Scytinium parvum (Degel.) Otálora, P.M. Jørg. \& Wedin

Scytinium pulvinatum (Hoffm.) Otálora, P.M. Jørg. \& Wedin

Scytinium schraderi (Bernh.) Otálora, P.M. Jørg. \& Wedin

Scytinium subtile (Schrad.) Otálora, P.M. Jørg. \& Wedin

Scytinium tenuissimum (Dicks.) Otálora,

P.M. Jørg. \& Wedin

Scytinium teretiusculum (Wallr.) Otálora,

P.M. Jørg. \& Wedin

Solorina bispora $\mathrm{Nyl}$.

Solorina saccata (L.) Ach.

Solorina spongiosa (Ach.) Anzi

Sphaerophorus globosus (Huds.) Vain.

Squamarina lentigera (Weber) Poelt

\begin{tabular}{|c|c|c|c|c|c|}
\hline $\begin{array}{l}\text { Species } \\
\text { name used } \\
\text { in } 2008\end{array}$ & $\begin{array}{l}\text { Cat- } \\
\text { egory } \\
2019\end{array}$ & Criteria 2019 & $\begin{array}{l}\text { Cat- } \\
\text { egory } \\
2008\end{array}$ & Criteria 2008 & $\begin{array}{l}\text { Com- } \\
\text { ment }\end{array}$ \\
\hline & EN & B2ab(iii); D & $\mathrm{DD}$ & & \\
\hline & VU & $\mathrm{D} 1+2$ & VU & D2 & \\
\hline & $\mathrm{CR}$ & $\mathrm{D}$ & $\mathrm{EN}$ & A2 & 1960 \\
\hline & $\mathrm{EN}$ & $\mathrm{A} 2 \mathrm{c}$ & NT & & III \\
\hline & $\mathrm{RE}$ & & RE & & 1932 \\
\hline & $\mathrm{RE}$ & & $\mathrm{RE}$ & & $<1890$ \\
\hline & $\mathrm{RE}$ & & $\mathrm{RE}$ & & 1929 \\
\hline $\begin{array}{l}\text { Collema } \\
\text { occultatum }\end{array}$ & CR & $\mathrm{D}$ & $\mathrm{RE}$ & & \\
\hline \multirow{6}{*}{$\begin{array}{l}\text { Xanthoria } \\
\text { sorediata }\end{array}$} & CR & $\mathrm{D}$ & VU & D2 & \\
\hline & $\mathrm{EN}$ & $\mathrm{D}$ & NT & & II \\
\hline & EN & $\mathrm{D}$ & VU & A3c; B2ab(iii) & II \\
\hline & NT & $\mathrm{C} 1$ & LC & & III \\
\hline & EN & $\mathrm{D}$ & VU & B2ab(iii) & II \\
\hline & $\mathrm{RE}$ & & $\mathrm{NE}$ & & 1935 \\
\hline \multirow{3}{*}{$\begin{array}{l}\text { Leptogium } \\
\text { gelatino- } \\
\text { sum }\end{array}$} & EN & $\mathrm{C} 2 \mathrm{a}(\mathrm{i}) ; \mathrm{D}$ & $\mathrm{DD}$ & & \\
\hline & NT & $\mathrm{A} 2 \mathrm{c}+4 \mathrm{c} ; \mathrm{C} 1 ; \mathrm{D}$ & $\mathrm{NE}$ & & \\
\hline & $\mathrm{EN}$ & $\mathrm{C} 2 \mathrm{a}(\mathrm{i}) ; \mathrm{D}$ & $\mathrm{NE}$ & & \\
\hline \multirow{2}{*}{$\begin{array}{l}\text { Collema } \\
\text { parvum }\end{array}$} & CR & $\mathrm{D}$ & VU & D2 & \\
\hline & NT & $\mathrm{A} 2 \mathrm{c}+4 \mathrm{c} ; \mathrm{C} 1 ; \mathrm{D}$ & $\mathrm{NE}$ & & \\
\hline $\begin{array}{l}\text { Leptogium } \\
\text { schraderi }\end{array}$ & EN & $\mathrm{C} 2 \mathrm{a}(\mathrm{i}) ; \mathrm{D}$ & VU & D2 & \\
\hline $\begin{array}{l}\text { Leptogium } \\
\text { subtile }\end{array}$ & CR & $\mathrm{C} 2 \mathrm{a}(\mathrm{i}) ; \mathrm{D}$ & VU & D2 & \\
\hline $\begin{array}{l}\text { Leptogium } \\
\text { tenuissi- } \\
\text { mum }\end{array}$ & $\mathrm{EN}$ & $\mathrm{C} 2 \mathrm{a}(\mathrm{i}) ; \mathrm{D}$ & $\mathrm{DD}$ & & \\
\hline \multirow{7}{*}{$\begin{array}{l}\text { Leptogium } \\
\text { teretiuscu- } \\
\text { lum }\end{array}$} & VU & $\mathrm{C} 1 ; \mathrm{D} 1$ & VU & B2ab(iii) & II \\
\hline & CR & $\begin{array}{l}\text { B1ab(iii)+2ab(iii); } \\
\text { D }\end{array}$ & VU & D2 & \\
\hline & $\mathrm{EN}$ & $\mathrm{C} 1$ & NT & & II \\
\hline & $\mathrm{EN}$ & $\begin{array}{l}\text { A2bc; } \\
\text { B1ab(iii)+2ab(iii); } \\
\text { D }\end{array}$ & EN & B1ab(iii) & II \\
\hline & CR & $\mathrm{D}$ & $\mathrm{CR}$ & B2ab(iii,iv) & \\
\hline & CR & $\mathrm{C} 2 \mathrm{a}(\mathrm{i}) ; \mathrm{D}$ & EN & B1ab(iii) & II \\
\hline & $\mathrm{EN}$ & $\mathrm{A} 2 \mathrm{c}$ & VU & $\mathrm{A} 2 \mathrm{c}$ & III \\
\hline
\end{tabular}

Stereocaulon condensatum Hoffm.
Leptogium EN C2a(i); D $\quad$ DD gelatino-

NT $\quad \mathrm{A} 2 \mathrm{c}+4 \mathrm{c} ; \mathrm{C} 1 ; \mathrm{D} \quad \mathrm{NE}$

EN C2a(i); D NE

NT $\mathrm{A} 2 \mathrm{c}+4 \mathrm{c} ; \mathrm{C} 1 ; \mathrm{D} \quad \mathrm{NE}$

Leptogium schraderi

Leptogium subtile

Leptogium tenuissi-

Leptogium teretiuscu-

B1ab(iii)+2ab(iii); VU

A2bc

B1 $\mathrm{ab}(\mathrm{iii})+2 \mathrm{ab}(\mathrm{iii})$;

EN B1ab(iii)

II

III 


\begin{tabular}{|c|c|c|c|c|c|c|}
\hline Species name & $\begin{array}{l}\text { Species } \\
\text { name used } \\
\text { in } 2008\end{array}$ & $\begin{array}{l}\text { Cat- } \\
\text { egory } \\
2019\end{array}$ & Criteria 2019 & $\begin{array}{l}\text { Cat- } \\
\text { egory } \\
2008\end{array}$ & Criteria 2008 & $\begin{array}{l}\text { Com- } \\
\text { ment }\end{array}$ \\
\hline Stereocaulon evolutum Graewe ex Th. Fr. & & $\mathrm{RE}$ & & $\mathrm{RE}$ & & 1908 \\
\hline Stereocaulon incrustatum Flörke & & CR & $\mathrm{C} 1+2 \mathrm{a}(\mathrm{i}) ; \mathrm{D}$ & DD & & \\
\hline Stereocaulon vesuvianum Pers. & & $\mathrm{RE}$ & & $\mathrm{RE}$ & & 1912 \\
\hline Zwackhia sorediifera (P. James) Ertz & $\begin{array}{l}\text { Opegrapha } \\
\text { soredifera }\end{array}$ & CR & $\mathrm{C} 2 \mathrm{a}(\mathrm{i}) ; \mathrm{D}$ & VU & D2 & \\
\hline Zwackhia viridis (Ach.) Poetsch \& Schied. & $\begin{array}{l}\text { Opegrapha } \\
\text { viridis }\end{array}$ & VU & $\mathrm{C} 1 ; \mathrm{D} 1$ & VU & $\mathrm{A} 3 \mathrm{c}$ & \\
\hline Thelidium pyrenophorum (Ach.) Mudd & & $\mathrm{DD}$ & & RE & & \\
\hline Thelotrema lepadinum (Ach.) Ach. & & VU & $\mathrm{C} 1$ & NT & & III \\
\hline Toninia physaroides (Opiz) Zahlbr. & & VU & C1; D1 & - & & \\
\hline Toninia sedifolia (Scop.) Timdal & & $\mathrm{EN}$ & $\begin{array}{l}\text { B1ab(iii) }+2 \mathrm{ab}(\mathrm{iii}) \\
\text { C1; D }\end{array}$ & VU & B2ab(iii) & \\
\hline Toninia verrucarioides ( $\mathrm{Nyl}$.) Timdal & & CR & $\begin{array}{l}\text { B1ab(iii) }+2 \mathrm{ab}(\mathrm{iii}) \\
\text { D }\end{array}$ & DD & & \\
\hline Umbilicaria cinerascens (Arnold) Frey & & CR & $\mathrm{D}$ & VU & D2 & \\
\hline Umbilicaria cylindrica (L.) Delise ex Duby & & CR & $\mathrm{D}$ & $\mathrm{DD}$ & & 1959 \\
\hline Umbilicaria decussata (Vill.) Zahlbr. & & CR & $\begin{array}{l}\text { B2ab(iv); C2a(i); } \\
\text { D }\end{array}$ & VU & D2 & \\
\hline Umbilicaria hirsuta (Sw. ex Westr.) Hoffm. & & CR & $\mathrm{D}$ & - & & \\
\hline Umbilicaria hyperborea (Ach.) Hoffm. & & $\mathrm{EN}$ & $\mathrm{D}$ & VU & D2 & \\
\hline Umbilicaria nylanderiana (Zahlbr.) H. Magn. & & CR & $\mathrm{C} 2 \mathrm{a}(\mathrm{i}) ; \mathrm{D}$ & VU & D2 & \\
\hline Umbilicaria polyrrhiza (L.) Fr. & & $\mathrm{EN}$ & $\mathrm{D}$ & CR & $\mathrm{A} 2 \mathrm{bc}$ & \\
\hline Umbilicaria proboscidea (L.) Schrad. & & CR & $\mathrm{D}$ & DD & & \\
\hline Usnea barbata (L.) Weber ex F.H. Wigg & & NT & $\mathrm{A} 2 \mathrm{c}+3 \mathrm{c}+4 \mathrm{c} ; \mathrm{C} 1$ & NT & & III \\
\hline Usnea chaetophora Stirt. & & EN & B1ab(iii); D & EN & B1ab(iii) & \\
\hline Usnea fulvoreagens (Räsänen) Räsänen & & VU & $\mathrm{A} 2 \mathrm{c} ; \mathrm{C} 1 ; \mathrm{D} 1$ & EN & $\mathrm{A} 2 \mathrm{bc}+3 \mathrm{bc}$ & \\
\hline Usnea glabrata (Ach.) Vain. & & $\mathrm{CR}$ & $\mathrm{A} 2 \mathrm{c} ; \mathrm{C} 2 \mathrm{a}(\mathrm{i})$ & CR & $\mathrm{A} 2 \mathrm{bc}+3 \mathrm{bc}$ & 1959 \\
\hline Usnea wasmuthii Räsänen & & VU & A3c; B2ab(iii) & VU & $\mathrm{A} 2 \mathrm{bc}+3 \mathrm{bc}$ & \\
\hline Vahliella leucophaea (Vahl) P.M. Jørg. & $\begin{array}{l}\text { Fuscopan- } \\
\text { naria leu- } \\
\text { cophaea }\end{array}$ & CR & $\begin{array}{l}\text { A2bc; B1ab(i,ii)+ } \\
\text { 2ab(i,ii); D }\end{array}$ & $\mathrm{DD}$ & & 1961 \\
\hline $\begin{array}{l}\text { Variospora thallincola (Wedd.) Arup, Frödén \& } \\
\text { Søchting }\end{array}$ & $\begin{array}{l}\text { Caloplaca } \\
\text { thallincola }\end{array}$ & EN & $\mathrm{D}$ & VU & D2 & \\
\hline Verrucaria maculiformis Kremp. & & DD & & $\mathrm{RE}$ & & \\
\hline $\begin{array}{l}\text { Vulpicida juniperinus (L.) J.-E. Mattsson \& } \\
\text { M. J. Lai }\end{array}$ & & NT & $\begin{array}{l}\text { A2c+3c+4c; } \\
\text { B1ab(iii) }\end{array}$ & $\mathrm{DD}$ & & \\
\hline Xanthomendoza fallax (Hepp) Arnold & & $\mathrm{EN}$ & $\mathrm{D}$ & VU & B1ab(i,iv) & \\
\hline $\begin{array}{l}\text { Xanthoparmelia mongeotii (Schaer. ex D. Dietr.) } \\
\text { Hale }\end{array}$ & & $\mathrm{EN}$ & $\mathrm{C} 2 \mathrm{a}(\mathrm{i})$ & $\mathrm{EN}$ & B2ab(iii) & II \\
\hline Xanthoria calcicola Oxner & & EN & $\mathrm{D}$ & VU & D2 & \\
\hline *Xanthoria oregana Gyeln. & & DD & & - & & \\
\hline
\end{tabular}

* as Gallowayella poeltii in Estonian Nature Information System 
Supplement 2. The list of lichen species which were red-listed in 2008, but considered as Not Evaluated (NE) or Not Applicable (NA) in 2019. Explanation why the species was not assessed in 2019 is given in the Comment.

\begin{tabular}{|c|c|c|c|}
\hline \multirow[t]{2}{*}{ Species name in 2008} & \multicolumn{2}{|c|}{ Red list category } & \multirow[t]{2}{*}{ Comment } \\
\hline & 2008 & 2019 & \\
\hline $\begin{array}{l}\text { Bryoria intricans (Vain.) } \\
\text { Brodo \& D. Hawksw. }\end{array}$ & DD & NA & $\begin{array}{l}\text { has been included in Bryoria chalybeiformis (L.) Brodo \& D. } \\
\text { Hawksw. and was assessed within this species in } 2019\end{array}$ \\
\hline $\begin{array}{l}\text { Caloplaca coronata (Kremp. } \\
\text { ex Körb.) J. Steiner }\end{array}$ & VU & NE & $\begin{array}{l}\text { Estonian material needs re-identification and therefore the species } \\
\text { was not assessed in } 2019\end{array}$ \\
\hline $\begin{array}{l}\text { Cladonia convoluta (Lam.) } \\
\text { Anders }\end{array}$ & VU & NA & $\begin{array}{l}\text { has been included in Cladonia foliacea (Huds.) Willd. and was as- } \\
\text { sessed within this species in } 2019\end{array}$ \\
\hline $\begin{array}{l}\text { Dermatocarpon leptophyllum } \\
\text { (Ach.) K.G.W. Lång }\end{array}$ & $\mathrm{DD}$ & NA & $\begin{array}{l}\text { has been included in Dermatocarpon miniatum (L.) W. Mann and } \\
\text { was assessed within this species in } 2019\end{array}$ \\
\hline $\begin{array}{l}\text { Hyperphyscia adglutinata } \\
\text { H. Mayrhofer \& Poelt }\end{array}$ & $\mathrm{RE}$ & NA & $\begin{array}{l}\text { considered doubtful (included in the checklist based on literature } \\
\text { data, herbarium samples are not available) and therefore the spe- } \\
\text { cies was not assessed in } 2019\end{array}$ \\
\hline Lecanora swartzii (Ach.) Ach. & VU & $\mathrm{NE}$ & $\begin{array}{l}\text { lack of data for the assessment (only one collection known from } \\
\text { Mohni island, 2005) }\end{array}$ \\
\hline $\begin{array}{l}\text { Leptogium cyanescens } \\
\text { (Rabenh.) Körb. }\end{array}$ & $\mathrm{DD}$ & NA & $\begin{array}{l}\text { considered doubtful (included in the checklist based on literature } \\
\text { data, herbarium samples are not available) and therefore the spe- } \\
\text { cies was not assessed in } 2019\end{array}$ \\
\hline Opegrapha rupestris Pers. & VU & NA & $\begin{array}{l}\text { Estonian material needs re-identification and therefore the species } \\
\text { was not assessed in } 2019\end{array}$ \\
\hline $\begin{array}{l}\text { Vulpicida tubulosus (Schaer.) } \\
\text { J.-E. Mattsson \& M.J. Lai }\end{array}$ & NT & NA & $\begin{array}{l}\text { has been included in } V \text {. juniperinus (L.) J.-E. Mattsson \& M.J. Lai } \\
\text { (Saag et al. 2014) and was assessed within this species in } 2019\end{array}$ \\
\hline Usnea diplotypus Vain. & NT & $\mathrm{NE}$ & $\begin{array}{l}\text { has been included in } U \text {. dasopoga Vain. (Clerc 2011), the species } \\
\text { which was assessed as LC in } 2008\end{array}$ \\
\hline Usnea substerilis Motyka & EN & $\mathrm{NE}$ & $\begin{array}{l}\text { has been included in U. lapponica Vain. (Mark et al. 2016), the } \\
\text { species which was assessed as LC in } 2008\end{array}$ \\
\hline
\end{tabular}

